\title{
Irregularly shaped lacunar infarction: risk factors and clinical significance
}

Infartos lacunares com morfologia irregular: fatores de risco e significado clínico

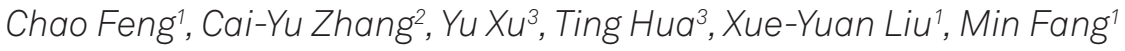

\begin{abstract}
Objective: Our study focused on acute lacunar infarct shapes to explore the risk factors and clinical significance of irregularly shaped lacunar infarctions. Methods: Based on the shape of their acute lacunar infarct, patients ( $n=204)$ were classified into the "regular" group or "irregular" group. The characteristics of the lacunar infarction were compared between the regular and irregular groups, between patients with and without neurological deterioration, and between patients with different modified Rankin scale (mRS) scores. The risk factors for irregularly shaped lacunar infarctions, neurological deterioration, and high mRS scores were identified. Results: Blood pressure variability (BPV) was an independent risk factor for irregularly shaped lacunar infarction. Infarction size, prevalence of advanced leukoaraiosis, and irregularly shaped lacunar infarcts were independent risk factors for higher mRS scores. Conclusions: The irregularly shaped lacunar infarcts were correlated with BPV. Irregularly shaped lacunar infarctions and leukoaraiosis may be associated with unfavorable clinical outcomes.
\end{abstract}

Keywords: blood pressure variability, lacunar infarction, lesion shape.

RESUMO

Objetivo: Estudar as diferentes formas dos infartos lacunares agudos, investigando os fatores de risco e o significado clinico daqueles com morfologia irregular. Métodos: Os 204 pacientes com infartos lacunares agudos foram classificados em dois grupos: aqueles com morfologia regular e aqueles com morfologia irregular. Foram estudadas as características dos dois grupos e caracterizados os fatores de risco para infartos irregulares, deterioração neurológica e altos escores da escala de Rankin modificada. Resultados: Variabilidade da pressão arterial é fator de risco independente para infartos lacunares irregulares. Tamanho do infarto, prevalência de leucoaraiose e formato irregular dos infartos lacunares são fatores de risco independentes para escores mais elevados na escala de Rankin modificada. Conclusões: Variabilidade da pressão arterial está relacionada ao formato irregular dos infartos lacunares agudos. Este tipo de infarto e a leucoaraiose podem estar relacionado a desfechos clínicos desfavoráveis.

Palavras-chave: formato de lesão, infarto lacunar, variabilidade da pressão arterial.

Lacunar infarcts are small infarcts with a diameter of 15-20 mm; they occur in the distributions of small penetrating arteries such as arteries in the thalamus, gangliocapsular regions, corona radiate, and brainstem ${ }^{1,2}$. The primary pathogenesis of small lacunar infarctions is the occlusion of small penetrating arteries resulting from hypertensive lipohyalinosis or fibroid necrosis ${ }^{2,3}$. Most lacunar infarcts appear as round, regular focal lesions, whereas other lacunar infarcts appear as irregularly shaped lesions ${ }^{4}$. Some lacunar infarcts even appear from the merging of several small lesions ${ }^{5}$.
Few studies have investigated the shapes of lacunar infarction; thus, little information is known about irregularly shaped lacunar infarctions such as their risk factors, pathological mechanisms, and clinical significance. A very interesting question is whether the irregular shape of a lacunar infarction is just a coincidence and has no clinical significance or whether the irregular shape represents a different pathogenesis from regularly shaped lacunar infarctions and has some influence on the clinical symptoms and prognosis of stroke. We hypothesized that lacunar strokes with irregularly

'Department of Neurology, Shanghai Tenth People's Hospital of Tongji University, Shanghai, China;

2Department of Neurology, Weihai Woman and Children's Hospital, Weihai, Shangdong, China;

${ }^{3}$ Department of Radiology, Shanghai Tenth People's Hospital of Tongji University, Shanghai, China.

Correspondence: Xue-Yuan Liu and Min Fang; Department of Neurology, Shanghai Tenth People's Hospital of Tongji University; Middle Yanchang Road Number 301 / Zhabei District; Shanghai, China; E-mail: liu1304@yeah.net (X.-Y. Liu); fangmin-dr@sohu.com (M. Fang)

Conflict of interest: There is no conflict of interest to declare.

Financial support: This study was supported by grants from the National Natural Science Foundation of China (No. 81000492 , No. 30971029 , and No. 81171163).

Received: 06 April 2013. Received in final form: 10 May 2013; Accepted: 17 May 2013. 
shaped infarcts have different pathogeneses, clinical symptoms, and prognoses in comparison to lacunar strokes with regularly shaped infarcts. In this study, we evaluated the risk factors, severity of neurological deficits, prognosis, and pathogeneses of irregularly shaped lacunar infarctions.

\section{METHODS}

\section{General information}

This study was based on consecutive patients who attended the Department of Neurology of Tenth People's Hospital (Shanghai, China) from January 2012 to September 2012 within the first 24 hours after stroke onset and were diagnosed as having an acute lacunar infarction. The identified infarcts had a diameter of less than $20 \mathrm{~mm}$ (as determined by magnetic resonance imaging [MRI]) and were categorized as small vessel occlusions, based on the Classification of Stroke System $^{6}$. Patients who underwent thrombolytic therapy, patients with previous physical disabilities, and patients who could not be followed up were excluded from the study. Two hundred four patients were enrolled in this study.

Patients were divided into two groups (i.e. the regular group and the irregular group), based on the shape of their infarctions, as determined by MRI. The shape of an infarction was considered "regular" when the infarction was ovoid, spheroid, or stick-like, and the shape of an infarction was considered "irregular" when the infarction was slab-like with multiple angles or was composed of multiple shapes or conglomerated beads ${ }^{4,5}$ (Figure).

The blood pressure was measured after the patients were admitted in the hospital. The ambulatory blood pressure was

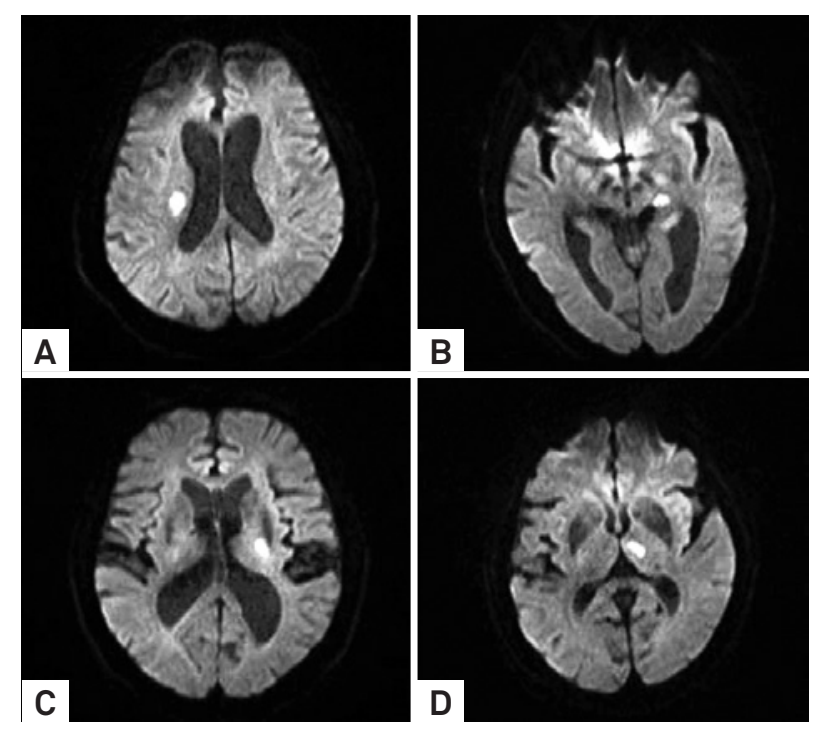

Figure. Regularly and irregularly shaped lacunar infarctions. (A and B) Irregularly shaped lacunar infarctions on diffusionweighted images (DWI). (C and D) Regularly shaped lacunar infarctions on DWI. monitored during the first 24 hours of hospitalization. The 24hour systolic blood pressure standard deviation (24hSBPSD) was recorded to indicate blood pressure variation (BPV). The following levels were tested: blood glucose, hemoglobin A1c (HbAlc), and blood lipids, which included triglyceride (TG), total cholesterol (TC), low-density lipoprotein (LDL), and high-density lipoprotein (HDL). On days 1 and 14 of hospitalization, the severity of neurological deficits was evaluated in accordance with the National Institute of Health stroke scale (NIHSS). An increased NIHSS score on day 14 was regarded as neurological deterioration. Before hospital discharge, the patients were administered individualized rehabilitation treatment plans by a rehabilitation therapist. After three months, a telephone interview was conducted by using a modified Rankin's scale (mRS) to evaluate the clinical outcomes of each patient. All procedures were approved by the Ethics Committee of the Shanghai Tenth People's Hospital of Tongji University (Shanghai, China) and were performed with the written consent of the patients if they had no impairment in cognitive function and were capable of writing or from the guardians of the patients if a patient had impaired consciousness, cognitive function, or writing ability.

\section{MR protocols}

Patients were imaged by using a 3.0T MRI scanner (Siemens 3.0T Magnetom Verio, Medical Solutions, Erlangen, Germany) with a standard eight-channel phasedarray head coil. T1-weighted images (repetition time/echo time $=2000 / 9$ ), T2-weighted images (repetition time/echo time $=6000 / 94)$, fluid-attenuated inversion recovery (FLAIR) images (repetition time/echo time=8500/94), and diffusionweighted images (DWI) (motion-probing gradients in three directions with a $b$ factor of $1000 \mathrm{~s} / \mathrm{mm}^{2}$ ) were obtained from the axial plane with a slice thickness of $5.5 \mathrm{~mm}$.

\section{MR images review and criteria}

Images were analyzed by two experienced radiologists who were blinded to the clinical information. An acute lacunar infarct was defined as a hypointense focal lesion with a diameter of 3-20 $\mathrm{mm}$ in T1-weighted images and as a hyperintense focal lesion in T2-weighted, FLAIR, and DWI images $^{1,2}$. The shape of the infarct was evaluated in accordance with the criteria described previously. The longest diameter of the infarcts was measured from DWIs. Leukoaraiosis was also evaluated from the FLAIR images and scored as grade 0 to grade 3, based on the Fazekas scale ${ }^{7}$. Grades 2 and 3 were regarded as evidence of advanced leukoaraiosis (ad-LA). The discrepancy of the shape of the infarct and leukoaraiosis score was resolved by visual consensus.

\section{Statistical analysis}

Data were analyzed by using SAS 9.2 software. The Chi square test and Student $t$ test were used to compare the risk 
factors between patients with regular infarctions and patients with irregular infarctions and between patients with neurological deterioration and patients without neurological deterioration. The analysis of variance (ANOVA) and Chi square test were used to compare risk factors of patients with different mRS scores. Logistic regression models were constructed to identify independent risk factors for irregular infarctions, neurological deterioration, and a high mRS score. A $\mathrm{p}<0.05$ indicated statistical difference.

\section{RESULTS}

In this study, 138 patients had regular infarctions and 66 patients had irregular infarctions. Compared to the patients in the regular group, the patients in the irregular group had a larger infarction size, higher diastolic blood pressure (DBP), and higher 24hSBPSD. The other factors did not show a statistical significance (Table 1). A logistic regression model was constructed to identify the possible risk factors of irregularly shaped lacunar infarctions. Information on age, sex, smoking, systolic blood pressure (SBP) and DBP after hospital admission, 24hSBPSD, HbAIc, TG, TC, LDL, and HDL were added to the model. The results showed that only $24 \mathrm{hSBPSD}$ is an independent risk factor for irregularly shaped lacunar infarctions $(\mathrm{OR}=1.64$; 95\% $\mathrm{CI}=1.26-21.4 ; \mathrm{p}<0.001)$.

Twenty (9.8\%) patients had neurological deterioration. The proportion of patients with irregularly shaped lacunar

Table 1. Baseline characteristics of patients with regular and irregular infarctions.

\begin{tabular}{lccc}
\hline & $\begin{array}{c}\text { Regular } \\
\text { group } \\
(\mathrm{n}=138)\end{array}$ & $\begin{array}{c}\text { Irregular } \\
\text { group } \\
(\mathrm{n}=66)\end{array}$ & $\mathrm{p}$ \\
\hline Age, years (SD) & $69.3(9.7)$ & $69.0(9.5)$ & 0.84 \\
Male, $\mathrm{n}(\%)$ & $72(52.2)$ & $43(65.2)$ & 0.08 \\
Smoking, $\mathrm{n}(\%)$ & $30(21.7)$ & $20(30.3)$ & 0.18 \\
Hypertension, $\mathrm{n}(\%)$ & $109(79.0)$ & $54(81.8)$ & 0.64 \\
Diabetes, $\mathrm{n}(\%)$ & $50(36.2)$ & $22(33.3)$ & 0.69 \\
Dyslipidemia, $\mathrm{n}(\%)$ & $95(68.8)$ & $46(69.7)$ & 0.90 \\
SBP, mmHg (SD) & $154.3(21.2)$ & $157.0(16.6)$ & 0.33 \\
DBP, mmHg (SD) & $84.3(11.8)$ & $88.8(9.4)$ & 0.003 \\
24hSBPSD, mmHg (SD) & $15.2(1.5)$ & $16.0(1.4)$ & $<0.001$ \\
HbA1c, \% (SD) & $6.2(1.1)$ & $6.2(1.3)$ & 0.92 \\
TG, mmol/L (SD) & $1.86(0.70)$ & $1.72(0.57)$ & 0.14 \\
TC, mmol/L (SD) & $5.05(1.09)$ & $4.83(0.85)$ & 0.13 \\
LDL, mmol/L (SD) & $3.36(0.88)$ & $3.20(0.72)$ & 0.21 \\
HDL, mmol/L (SD) & $1.04(0.23)$ & $1.09(0.27)$ & 0.20 \\
Diameter, mm (SD) & $1.06(0.28)$ & $1.33(0.24)$ & $<0.001$ \\
Ad-LA, $n$ (\%) & $82(59.4)$ & $48(72.7)$ & 0.06 \\
\hline 24hSBPD: 24-hour systol & &
\end{tabular}

24hSBPSD: 24-hour systolic blood pressure standard deviation; AdLA: advanced leukoaraiosis; DBP: diastolic blood pressure; HbAlc: hemoglobin Alc; HDL: high-density lipoprotein; LDL: low-density lipoprotein; SBP: systolic blood pressure; SBPSD: systolic blood pressure standard deviation; SD: standard deviation; TC: total cholesterol;TG: triglyceride. infarctions was higher in patients with neurological deterioration than in patients without neurological deterioration $(\mathrm{p}<0.01)$. Logistic regression was used to identify possible risk factors for neurological deterioration. Age, sex, smoking, size of infarct, prevalence of hypertension, diabetes, dyslipidemia, ad-LA, and irregularly shaped lacunar infarctions were added to the model. After adjusting for confounding factors, irregularly shaped lacunar infarction was identified as an independent risk factor for neurological deterioration $(\mathrm{OR}=4.54$, 95\%CI=1.42-14.58, $\mathrm{p}=0.01$ ).

Early prognosis of lacunar infarction was evaluated with the mRS. During the three-month follow up, no patients died or showed severe physical disabilities, required constant nursing care and attention, or became bedridden and incontinent. Thus, the mRS score ranged from 0 to 4 . The ANOVA results showed that age, prevalence of diabetes, ad-LA, irregular infarctions, SBP, HbAIc, and infarct size differed among patients with different mRS scores $(\mathrm{p}<0.05)$ (Table 2). Logistic regression was used to identify the risk factors for higher mRS scores. Age, sex, smoking, size of infarct, prevalence of hypertension, diabetes, dyslipidemia, ad-LA, and irregularly shaped lacunar infarction were added to the model. The results showed that infarct size, prevalence of ad-LA, and irregular infarction were independent risk factors for higher mRS scores (Table 3).

\section{DISCUSSION}

The present study focused on the shape of lacunar infarcts and analyzed the risk factors and clinical features of irregularly shaped lacunar infarctions. The following results were obtained: (1) irregularly shaped lacunar infarctions were prevalent in $32.3 \%$ of the sample population; (2) the $24 \mathrm{hSBPSD}$ is the only independent risk factor for irregularly shaped lacunar infarction; (3) the irregular shape of a lacunar infarction is associated with a larger infarction size, neurological deterioration, and unfavorable early prognosis; and (4) leukoaraiosis is associated with an unfavorable early prognosis of lacunar infarction.

A recent study on the shape of acute lacunar infarcts reports that $32.4 \%$ of these infarcts are conglomerated beadshaped infarcts ${ }^{5}$. In the current study, the range of irregularly shaped infarcts was greater than the range of conglomerated bead-shaped infarcts. However, the proportion of irregularly shaped infarcts in this study was not higher than the reported proportion of conglomerated bead-shaped infarcts. The relatively obscure standard used in the evaluation of the shape of infarcts may be the primary reason for the observed similarities in proportion. To date, no definite standard exists for classifying the shapes of infarcts. Thus, visual consensus used in different studies remains the only method of classifying infarcts on the basis of their shape. 
Table 2. Characteristics of patients at different mRS scores.

\begin{tabular}{|c|c|c|c|c|c|c|}
\hline & $\begin{array}{c}\mathrm{mRS}=0 \\
(n=39)\end{array}$ & $\begin{array}{r}\mathrm{mRS}=1 \\
(n=83)\end{array}$ & $\begin{array}{c}\mathrm{mRS}=2 \\
(\mathrm{n}=46)\end{array}$ & $\begin{array}{c}\mathrm{mRS}=3 \\
(\mathrm{n}=21)\end{array}$ & $\begin{array}{c}\mathrm{mRS}=4 \\
(n=15)\end{array}$ & $p$ \\
\hline Age, years (SD) & $65.7 \pm 9.4$ & $68.8 \pm 9.5$ & $70.4 \pm 9.5$ & $70.7 \pm 9.7$ & $74.4 \pm 8.2$ & 0.03 \\
\hline Male, n (\%) & $21(53.9)$ & $48(57.8)$ & $27(58.7)$ & $14(66.7)$ & 5 (33.3) & 0.36 \\
\hline Smoking, n (\%) & $8(20.5)$ & $22(26.5)$ & $11(23.9)$ & $8(38.1)$ & $1(6.7)$ & 0.27 \\
\hline Hypertension, n (\%) & $32(82.1)$ & $62(74.7)$ & $38(82.6)$ & $16(76.2)$ & $15(100.0)$ & 0.22 \\
\hline Diabetes, n (\%) & $7(18.0)$ & $33(39.8)$ & $18(39.1)$ & $5(23.8)$ & $9(60.0)$ & 0.02 \\
\hline Dyslipidemia, n (\%) & $30(76.9)$ & $58(69.9)$ & $31(67.4)$ & $12(57.1)$ & $10(66.7)$ & 0.62 \\
\hline SBP, mmHg (mean $\pm S D)$ & $158.1 \pm 20.3$ & $152.2 \pm 21.1$ & $154.2 \pm 16.3$ & $153.5 \pm 22.2$ & $169.4 \pm 11.8$ & 0.03 \\
\hline DBP, mmHg (mean $\pm S D)$ & $82.8 \pm 10.5$ & $84.7 \pm 12.0$ & $88.9 \pm 9.3$ & $85.9 \pm 13.5$ & $89.3 \pm 9.4$ & 0.07 \\
\hline $\begin{array}{l}\text { 24hSBPSD, mmHg } \\
(m e a n \pm S D)\end{array}$ & $15.2 \pm 1.4$ & $15.6 \pm 1.5$ & $15.6 \pm 1.5$ & $15.1 \pm 1.9$ & $15.4 \pm 1.3$ & 0.50 \\
\hline 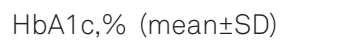 & $5.7 \pm 0.7$ & $6.2 \pm 1.2$ & $6.3 \pm 1.2$ & $6.1 \pm 1.4$ & $6.7 \pm 1.7$ & 0.04 \\
\hline LDL, mmol/L (mean $\pm S D)$ & $3.36 \pm 0.79$ & $3.39 \pm 0.84$ & $3.25 \pm 0.86$ & $3.20 \pm 0.80$ & $3.06 \pm 0.89$ & 0.59 \\
\hline Diameter, mm (mean $\pm S D)$ & $1.12 \pm 0.29$ & $1.09 \pm 0.25$ & $1.26 \pm 0.30$ & $1.27 \pm 0.30$ & $1.30 \pm 0.34$ & $<0.001$ \\
\hline Ad-LA, n (\%) & $13(33.3)$ & $54(65.1)$ & $32(69.6)$ & $17(81.0)$ & $14(93.3)$ & $<0.001$ \\
\hline Irregular shape, n (\%) & $2(5.1)$ & $19(22.9)$ & $28(60.9)$ & $9(42.9)$ & $8(53.3)$ & $<0.001$ \\
\hline
\end{tabular}

24hSBPSD: 24-hour systolic blood pressure standard deviation; AdLA: advanced leukoaraiosis; DBP: diastolic blood pressure; HbAlc: hemoglobin Alc; LDL: low-density lipoprotein; mRS: modified Rankin Scale; SBP: systolic blood pressure; SD: standard deviation.

Table 3. Risk factors for the higher mRS scores.

\begin{tabular}{lccc}
\hline & OR & $95 \% \mathrm{Cl}$ & $\mathrm{p}$ \\
\hline Diameter, +1 mm & 4.34 & $1.57-12.0$ & 0.005 \\
Ad-LA (1 vs. 0) & 2.41 & $1.15-5.05$ & 0.02 \\
Irregular shape (1 vs. 0) & 3.28 & $1.74-6.18$ & $<0.001$ \\
\hline
\end{tabular}

AdLA: advanced leukoaraiosis; $\mathrm{Cl}$ : confidence interval; OR: odds ratio.

Hypertension is a primary independent risk factor for lacunar infarctions, including silent and acute infarctions ${ }^{2}$. Hypertension, particularly longstanding hypertension, is the primary reason for lipohyalinosis and fibroid necrosis, which occlude the small arteries and thus result in lacunar infarctions, especially infarcts with diameters less than $15-20 \mathrm{~mm}^{1}$. In this study, most infarctions were associated with hypertension. Similar prevalence rates of hypertension, SBP, and DBP were found in patients with regular and irregular infarctions. However, BPV was more significant in patients with irregular infarctions. Blood pressure variability has been associated with an increased risk of stroke ${ }^{8,9}$. This study showed that BPV is also associated with irregularly shaped lacunar infarctions. Clinical and experimental studies prove that longstanding hypertension with significant BPV may cause severe endothelial dysfunction of the small blood vessels ${ }^{10,11}$, thereby resulting in the breakage of the blood-brain barrier and enlargement of Virchow-Robin spaces ${ }^{12}$. Therefore, BPV may be associated with the severity of small vessel disease (SVD) such as the severity of leukoaraiosis ${ }^{13,14}$. In this study, the severity of leukoaraiosis showed a weak association with irregular infarctions $(\mathrm{p}=0.06)$.

Leukoaraiosis reduces vascular density ${ }^{15}$ and cerebral blood flow ${ }^{16}$, and may result in infarct growth by preventing peripheral compensation during ischemic stress ${ }^{17}$. This mechanism may be associated with the pathogenesis of irregularly shaped infarcts, which had larger sizes than regular infarcts in this study. The irregular shape of infarcts may be caused by the asymmetric enlargement of the infarction. However, an intrinsic SVD-related mechanism may be more significant in irregular infarctions than in regular infarctions.

Previous studies prove a correlation between leukoaraiosis and unfavorable clinical outcomes of ischemic stro$\mathrm{ke}^{18}$. This association may be related to a dysfunctional neuronal network ${ }^{19}$ and enlarged infarction size due to leukoaraiosis ${ }^{17,18}$. In this study, a similar correlation was observed between irregularly shaped lacunar infarctions and clinical outcomes. Neurological deterioration on day 14 and outcomes after 3 months were independent of leukoaraiosis and infarct size. It is reasonable to assume that a large infarction would be associated with unfavorable clinical outcomes. However, the association between the shape of an infarct and clinical outcome remains puzzling. Because of infarction enlargement, it is possible that the size of the infarct in irregularly shaped infarctions may be even larger after 1 or 2 weeks than the shape determined via MRI within 24 hours after stroke onset. Other factors, such as inflammation ${ }^{20}$, which was not considered in this study, may also be related to this phenomenon.

The primary strengths of this study include its prospective study design, use of various statistical methods, and use of 24-hour ambulatory blood pressure monitoring that could supply accurate BPV. The primary limitations of this study include the limited sample size and non-specific standards for identifying different infarct shapes, which may be influenced by subjective judgments. Studies with larger sample sizes are required for further exploration of infarct shapes. 
1. Fisher CM. Lacunes: small, deep cerebral infarcts. Neurology 1965;15:774-784.

2. Moran C, Phan TG, Srikanth VK. Cerebral small vessel disease: a review of clinical, radiological, and histopathological phenotypes. Int J Stroke 2012;7:36-46.

3. Pantoni L. Cerebral small vessel disease: from pathogenesis and clinical characteristics to therapeutic challenges. Lancet Neurol 2010;9:689-701.

4. Herve D. Shape and volume of lacunar infarcts: a 3D MRI study in cerebral autosomal dominant arteriopathy with subcortical infarcts and leukoencephalopathy. Stroke 2005;36:2384-2388.

5. Ryu DW, Shon YM, Kim BS, Cho AH. Conglomerated beads shape of lacunar infarcts on diffusion-weighted MRI: what does it suggest? Neurology 2012;78:1416-1419.

6. Ay H, BennerT, Arsava EM, et al. A computerized algorithm for etiologic classification of ischemic stroke: the Causative Classification of Stroke System. Stroke 2007;38:2979-2984.

7. Fazekas F, Chawluk JB, Alavi A, Hurtig HI, Zimmerman RA. MR signal abnormalities at $1.5 \mathrm{~T}$ in Alzheimer's dementia and normal aging. AJR 1987;149:351-356.

8. Shimbo D, Newman JD, Aragaki AK, et al. Association between annual visit-to-visit blood pressure variability and stroke in postmenopausal women: data from the Women's Health Initiative. Hypertension 2012;60:625-630.

9. Rothwell PM, Howard SC, Dolan E, et al. Prognostic significance of visit-to-visit variability, maximum systolic blood pressure, and episodic hypertension. Lancet 2010;375:895-905.

10. Diaz KM, Veerabhadrappa P, Kashem MA, et al. Relationship of visit-to-visit and ambulatory blood pressure variability to vascular function in African Americans. Hypertens Res 2012;35:55-61.
11. Eto M, Toba K, Akishita M, et al. Reduced endothelial vasomotor function and enhanced neointimal formation after vascular injury in a rat model of blood pressure lability. Hypertens Res 2003;26:991-998.

12. Klarenbeek P, van Oostenbrugge RJ, Lodder J, Rouhl RP, Knottnerus IL, Staals J. Higher ambulatory blood pressure relates to enlarged Virchow-Robin spaces in first-ever lacunar stroke patients. J Neurol 2013;260:115-121.

13. Gunstad J, Cohen RA, Tate DF, et al. Blood pressure variability and white matter hyperintensities in older adults with cardiovascular disease. Blood Press 2005;14:353-358.

14. Xiong $Y Y$, Mok V. Age-related white matter changes. J Aging Res 2011;201:1-13.

15. Moody DM, Thore CR, Anstrom JA, Challa VR, Langefeld CD, Brown WR. Quantification of afferent vessels shows reduced brain vascular density in subjects with leukoaraiosis. Radiology 2004;233:883-890.

16. O'Sullivan M, Lythgoe DJ, Pereira AC, et al. Patterns of cerebral blood flow reduction in patients with ischemic leukoaraiosis. Neurology 2002;59:321-326.

17. Heistad DD, Mayhan WG, Coyle P, Baumbach GL. Impaired dilatation of cerebral arterioles in chronic hypertension. Blood Vessels 1990;27:258-262.

18. Arsava EM, Rahman R, Rosand J, et al. Severity of leukoaraiosis correlates with clinical outcome after ischemic stroke. Neurology 2009;72:1403-1410.

19. Yamanouchi $H$, Sugiura S, Tomonaga M. Decrease in nerve fibres in cerebral white matter in progressive subcortical vascular encephalopathy of Binswanger type. An electron microscopic study. J Neurol 1989;236:382-387.

20. Tuttolomondo A, Di Raimondo D, Pecoraro R, Arnao V, Pinto A, Licata G. Inflammation in ischemic stroke subtypes. Curr Pharm Des 2012;18:4289-4310. 